Ann. Biol. anim. Bioch. Biophys., I973, 13 (4), 659-68ז.

\title{
CONSTRUCTION ET FONCTIONNEMENT DE 2 CHAMBRES RESPIRATOIRES DU TYPE « CIRCUIT OUVERT » POUR JEUNES BOVINS
}

\author{
M. VERMOREL, J.-C. BOUVIER, Y. BONNET et G. FAUCONNEAU \\ avec la collaboration technique de M. Dondainas \\ Station d'Étude des Métabolismes, \\ Centre de Recherches de Clermont-Ferrand, I. N. R. A., \\ Theix, 63110 Beaumont
}

\section{RÉSUMÉ,}

Les 2 chambres respiratoires du type "circuit ouvert " décrites sont destinées à des études de nutrition et de bioclimatologie avec des jeunes bovins pesant jusqu'à $350 \mathrm{~kg}$ et des groupes d'agneaux. Le système de climatisation contrôle la température et l'humidité relative sur une large plage. Les débitmètres et les analyseurs de gaz assurent une mesure très précise des échanges respiratoires de l'animal. L'air est analysé en continu; de plus, un dispositif de secours permet de constituer un prélèvement d'air représentatif sur une période de 24 heures.

L'installation est équipée de capteurs physiques (températures, vitesse de l'air) et physiologiques (températures, fréquences cardiaque et respiratoire), de dispositifs de sécurité et d'alarme. Les différents capteurs sont scrutés par une centrale de mesure avec une périodicité variable. Les données sont imprimées sur papier et perforées sur ruban en vue du traitement mécanographique. Les mesures sont réalisées pendant 4 jours consécutifs, après 2 jours d'adaptation, avec une précision élevée : la différence entre le bilan calorique et le bilan carbone-azote est égal à $0,2 \pm \mathrm{I}, 2 \mathrm{p}$. Ioo de l'énergie ingérée. Cette installation offre de nombreuses possibilités de travail, en particulier l'étude des variations du métabolisme énergétique au cours du cycle nycthéméral.

\section{I. - INTRODUCTION}

L'intérêt de la calorimétrie indirecte par rapport à la méthode des abattages comparés est de permettre de déterminer sur une période de courte durée (I à quelques jours) plusieurs fois au cours de la vie du même animal l'énergie fixée, totale, sous forme de protéines et de lipides, dans des conditions physiologiques, nutritionnelles, climatiques bien définies mais variables et successives. Cet avantage est parti- 
culièrement appréciable pour des études sur les animaux en croissance. Cette méthode permet d'obtenir, à partir du quotient respiratoire, certaines indications sur le métabolisme des nutriments. Elle se prête également aux études de bioclimatologie pour la détermination de la production de chaleur de l'animal placé dans diverses conditions climatiques, variables au cours du cycle nycthéméral.

Les installations du type "circuit fermé " ont été beaucoup utilisées pour les études d'alimentation et de nutrition (WAINMAN et BLAXTER, I958; AlEXANDER, I96I ; GrAHAM, I962). De réalisation relativement simple, elles permettent d'obtenir des bilans énergétiques précis sur une période de 24 heures. Elles présentent, pour les études sur ruminants l'inconvénient de l'accumulation de méthane dans les chambres respiratoires. Dans le système du " circuit ouvert ", cet inconvénient est évité puisque la chambre respiratoire est balayée en permanence par de l'air frais. Une analyse continue de l'air qui sort de la chambre permet de suivre l'évolution des échanges respiratoires de l'animal, condition nécessaire pour les études métaboliques et bioclimatologiques. La précision des résultats est le facteur limitant de cette méthode; elle est liée essentiellement aux caractéristiques des débitmètres et des analyseurs de gaz.

Les études que nous entreprenons sur des animaux en croissance sont variées et concernent principalement : la valeur énergétique nette de certains aliments, l'utilisation métabolique de nutriments particuliers, les variations de la composition du croît de l'animal avec la race, la souche, le régime et l'influence des conditions climatiques sur la croissance du veau de boucherie.

La nature de certaines de ces études impliquait l'utilisation d'installations du type " circuit ouvert", perfectionnées et d'un emploi souple, pourvues d'un équipement climatique puissant, d'un enregistrement continu des informations permettant un traitement mécanographique.

\section{II. - DESCRIPTION DES INSTALLATIONS}

\section{A. - Les chambres respiratoires}

Elles ont été conçues pour des jeunes bovins pesant jusqu'à $350 \mathrm{~kg}$ et construites suivant nos directives par la Société "La Physique appliquée ", 95 Taverny. Elles sont installées dans une pièce conditionnée en température. Les 2 caissons symétriques, de $2,22 \mathrm{~m}$ de longueur, $\mathrm{I}, 00 \mathrm{~m}$ de largeur et $\mathrm{I}, 68 \mathrm{~m}$ de hauteur intérieures (soit $3,73 \mathrm{~m}^{3}$ et $4,28 \mathrm{~m}^{3}$ avec les gaines) sont séparés par un sas de $\mathrm{I}, 08 \mathrm{~m}^{3}$ (fig. $\mathrm{I}$ ). Ces caissons reposent à $\mathrm{I} 7 \mathrm{~cm}$ du sol sur un bâti en acier profilé et des pieds réglables. Ils sont constitués de feuilles d'acier inoxydable de $3 \mathrm{~mm}$ d'épaisseur, soudées à l'argon et sont recouverts d'une couche de laine de verre de $5 \mathrm{~cm}$ et enveloppés d'une tôle d'acier peinte.

A l'arrière de chaque chambre une large porte $(82 \times 168 \mathrm{~cm})$ assure le passage des cages ou des animaux. Sur le côté, un portillon du sas permet de pénétrer dans les chambres en cours d'expérience sans fausser les mesures (VAN Es, I96I). A l'avant une petite porte est prévue pour l'alimentation des animaux chaque matin sans les déranger. Un large hublot $(100 \times 80 \mathrm{~cm})$ en plexiglass (Io $\mathrm{mm})$ situé à la partie 
antérieure de la chambre permet aux animaux de se voir et facilite leur adaptation. Des ronds de gants surmontés de 2 hublots de $50 \times 30 \mathrm{~cm}$, sur un côté et sur la porte arrière, facilitent les interventions depuis l'extérieur. A la partie postérieure se trouve

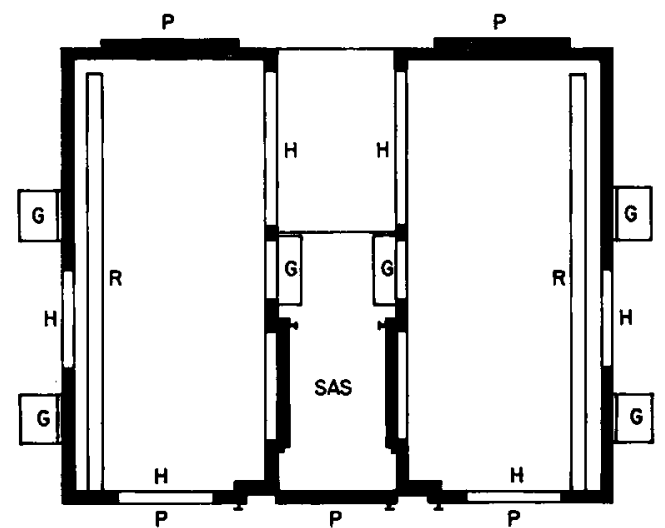

FIG. I. -- Schéma des 2 chambres respiratoires avec le sas (coupe par un plan horizontal)

$\mathrm{P}$ : porte ; $\mathrm{H}$ : hublot ; $\mathrm{G}$ : gaine d'aspiration ; $\mathrm{R}$ : rail

FIG. I. - Plan of the two respiration chambers with the airlock

$P$ : door ; $H$ : porthole; $G$ : ventilation shafts; $R$ : rail

un passage étanche pour 40 conducteurs électriques. Les animaux sont installés soit sur un plancher soit dans une cage métallique montée sur roulettes dont 2 sont guidées par un rail.

\section{B. - La climatisation}

Eille est indépendante dans les 2 chambres pour permettre de travailler dans des conditions différentes au cours des études de bioclimatologie. Les conditions climatiques demandées étaient, pour la température de +10 à $+30^{\circ} \mathrm{C}\left( \pm 0,5^{\circ} \mathrm{C}\right)$ et pour l'humidité relative 40 à $90 \mathrm{p}$. I00 ( $\pm 5 \mathrm{p}$. IOO). Nous nous sommes inspirés des installations de THORBEK (I969) pour la conception du système de climatisation.

I e conditionnement en température et en humidité relative est obtenu par le passage continu de 1'air sur une batterie froide fixant le point rosée recherché puis sur une batterie chaude ramenant l'air à la température fixée (fig. 2). Un ventilateur $\left(800 \mathrm{~m}^{3} / \mathrm{h}\right)$ reprend l'air à la base de la chambre respiratoire par 3 gaines équipées de filtres, le fait circuler sur les batteries d'échangeurs à raison de 200 renouvellements par heure et le ramène dans la chambre, au niveau du plafond, par l'intermédiaire d'un diffuseur en forme de calotte sphérique. L'introduction d'air frais se situe dans les gaines d'aspiration c'est-à-dire en amont des échangeurs. Chaque chambre est pourvue de 2 régulateurs de température BRM, l'un pour la température sèche $\left(t_{\mathrm{s}}\right)$, l'autre pour la température humide $\left(t_{\mathrm{n}}\right)$.

Pour l'obtention des hygrométries élevées aux températures extrêmes, une injection de vapeur entre les 2 échangeurs est nécessaire et réalisée par une chaudière assurant une production de 4 litres par heure. Nous réglons manuellement l'injection 
de vapeur pour chaque chambre et l'excédent est condensé sur la batterie froide. Un psychromètre est installé à la partie antérieure de la chambre, au niveau de la bouche d'extraction; il est composé de 2 sondes platines Ioo ohms et d'une turbine qui assure une vitesse d'air de $4 \mathrm{~m} /$ seconde au niveau des sondes.

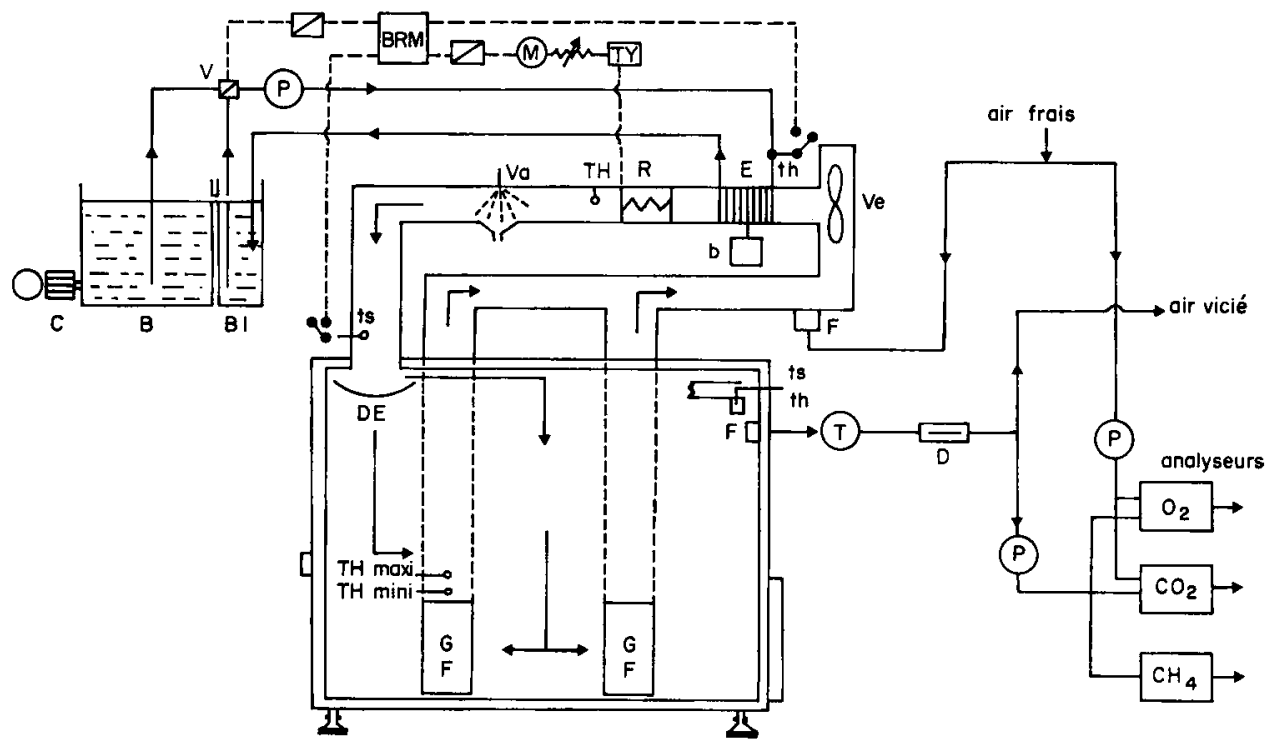

FIG. 2. - Schéma du système de climatisation et du circuit de l'air

B : bac à liquide réfrigérant (2 ooo 1) commun aux 2 chambres;

BI : bac intermédiaire ;

C : 2 compresseurs frigorifiques $(6600 \mathrm{kcal} / \mathrm{h}$ $\times 2)$;

$\mathrm{V}$ : vanne mélangeuse motorisée;

$\mathrm{P}$ : pompe à eau ou à air ;

$\mathrm{E}$ : échangeur froid à ailettes (30 $\mathrm{m}^{2}, \mathrm{~K}=\mathrm{I} 2 \mathrm{kcal} / \mathrm{h} / \mathrm{m}^{2} /{ }^{\circ} \mathrm{C}$ ) ;

$\mathbf{R}$ : résistances électriques $(\mathbf{I} \mathbf{k w} \times 8)$;

$\mathrm{Va}$ : injection de vapeur;

$\mathrm{DE}$ : déflecteur;
Ve : ventilateur;

b : bac à eau condensée, amovible.

F : filtres;

$\mathrm{G}$ : gaines;

$T$ : turbine d'extraction;

$\mathrm{D}$ : débitmètre ;

TH : thermostat de securité

ts et th : sondes de température seche et humide;

BRM : régulateur électronique double BRM ;

E : relais temporisateur (Is toutes les 40s);

$\mathrm{M}$ : potentiomètre ( $\mathrm{r}$ à $6 \mathrm{~V}$ ) ;

TY : bloc tyristor BTH $(0-220 \mathrm{~V})$.

FIG. 2. - Air conditioning system and ventilation circuit

B : tank of liquid refrigerant ( 2 ooo 1 ) common to both chambers;

$\mathrm{BI}$ : intermediate tank;

C: 2 refrigeration compressors $(6600 \mathrm{kcal} / \mathrm{h}$ $\times 2)$;

$\mathrm{V}$ : mixing ;

$P$ : air or water pump;

$\mathrm{E}:$ cooling plates $\left(30 \mathrm{~m}^{2}, \mathrm{~K}=\mathrm{I} 2 \mathrm{kcal} / \mathrm{h} / \mathrm{m}^{2} /{ }^{\circ} \mathrm{C}\right)$;

$\mathrm{R}$ : electric heating elements ( $\mathrm{kw} \times 8$ );

$\mathrm{Va}$ : vapouriser;

$\mathrm{DE}$ : deflector;
$\mathrm{Ve}$ : ventilation fan;

b : condensate tank, removable.

$\mathrm{F}$ : filters;

G : inlets ;

T . extraction fan;

TH : safety thermostat;

ts and th : dry and wet bulb temperature sensors;

BRM : electronic temperature regulator;

$\mathrm{F}_{\mathrm{M}}$ : delay relay (Is every $40 \mathrm{OS}$ );

TY : block thyristor BTH (0-220 V).

\section{C. - La ventilation}

La ventilation de la chambre respiratoire est assurée par l'extraction permanente d'air vicié, compensée par l'introduction d'air pris dans le local ventilé. Une turbine multi-étages (73I SRA or E.T.R.I., Paris) assure, compte tenu des pertes de 
charge dans le débitmètre, un débit stable (écart maximum 501 en $24 \mathrm{~h}$ ) que l'on peut régler entre 4 et $\mathrm{I}_{2} \mathrm{~m}^{3} / \mathrm{h}$, au moyen de rondelles de différents diamètres intérieurs placées à la sortie de la turbine.

\section{D. - Le débitmètre (Setaram, Lyon)}

Le débitmètre est un Venturi équipé d'un microdébitmètre thermique massique U 55 monté en dérivation (fig. 3). Ce dernier fournit un signal électrique linéarisé (o à $50 \mathrm{mV}$ ) proportionnel au débit volumique ramené à $0^{\circ} \mathrm{C}$ et $760 \mathrm{~mm} \mathrm{Hg}$. Le débit mesuré est donc indépendant de la température et de la pression atmosphérique. En revanche, le microdébitmètre thermique est sensible à la vapeur d'eau dont la capacité calorifique $(0,46 \mathrm{kcal} / \mathrm{kg})$ est différente de celle de l'air sec $(0,24 \mathrm{kcal} / \mathrm{kg})$; le calcul montre que la présence de vapeur d'eau entraîne une erreur de l'ordre de o,3 p. Ioo sur la mesure du débit.

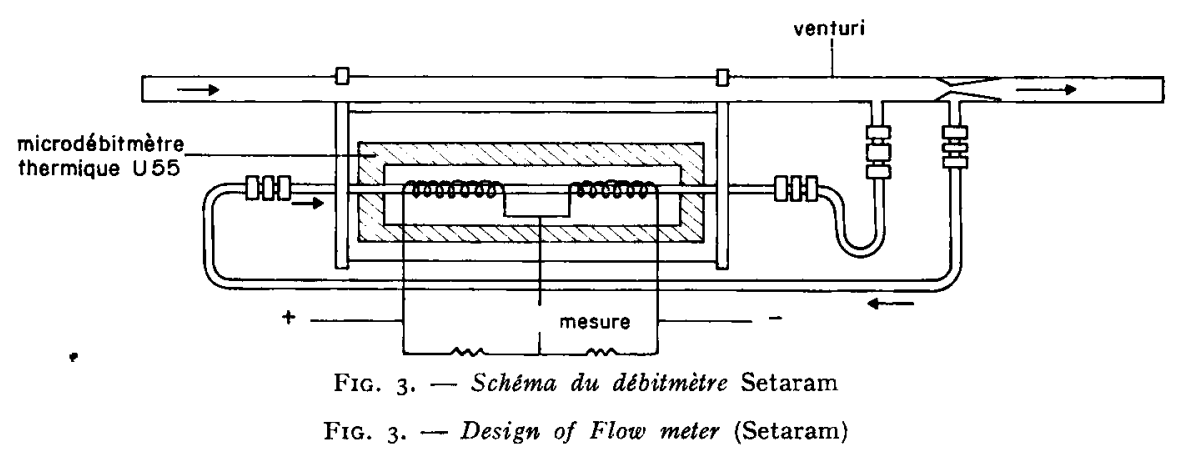

I1 est nécessaire de connaître la pression partielle de vapeur d'eau dans l'air puisque 1'analyse des gaz se fait sur un air partiellement ou totalement déshydraté et nous utilisons à cet effet les données du psychromètre placé dans la chambre respiratoire.

\section{E. - Les analyseurs de gaz}

Il ont été fournis par la Société Schlumberger (Massy). La baie d'analyse est située dans une pièce conditionnée en température; elle est symétrique et chaque partie correspond à une chambre respiratoire. Outre les analyseurs de gaz (oxygène, gaz carbonique, méthane), elle comporte l'équipement destiné au traitement de 1'air (fig. 4) : filtration, déshydratation, régulation de pression, avec un débit de $2501 /$ heure environ.

I. Les analyseurs de $\mathrm{CO}_{2}$, modèle Unor (Mahiak, Allemagne), sont basés sur le principe de l'absorption d'un rayonnement infrarouge par le gaz carbonique (SCHNEIDER, 1958). L'air qui entre dans les chambres respiratoires circule dans la cellule de référence, de sorte que l'analyseur indique directement la différence de composition de l'air (entre o et I p. Ioo) au cours de son passage dans la chambre respiratoire. Ces appareils sont très stables et peu sensibles à la vapeur d'eau mais très influencés par les variations de pression. 
2. L'analyseur de méthane, modèle Anir II (Schlumberger), comme les analyseurs de $\mathrm{CO}_{2}$, repose sur le principe de l'absorption d'un rayonnement infrarouge. I1 s'agit d'un appareil non différentiel équipé d'un filtre à $\mathrm{CO}_{2}$ dont l'échelle de mesure est de $o$ à $0, I$ p. Ioo.

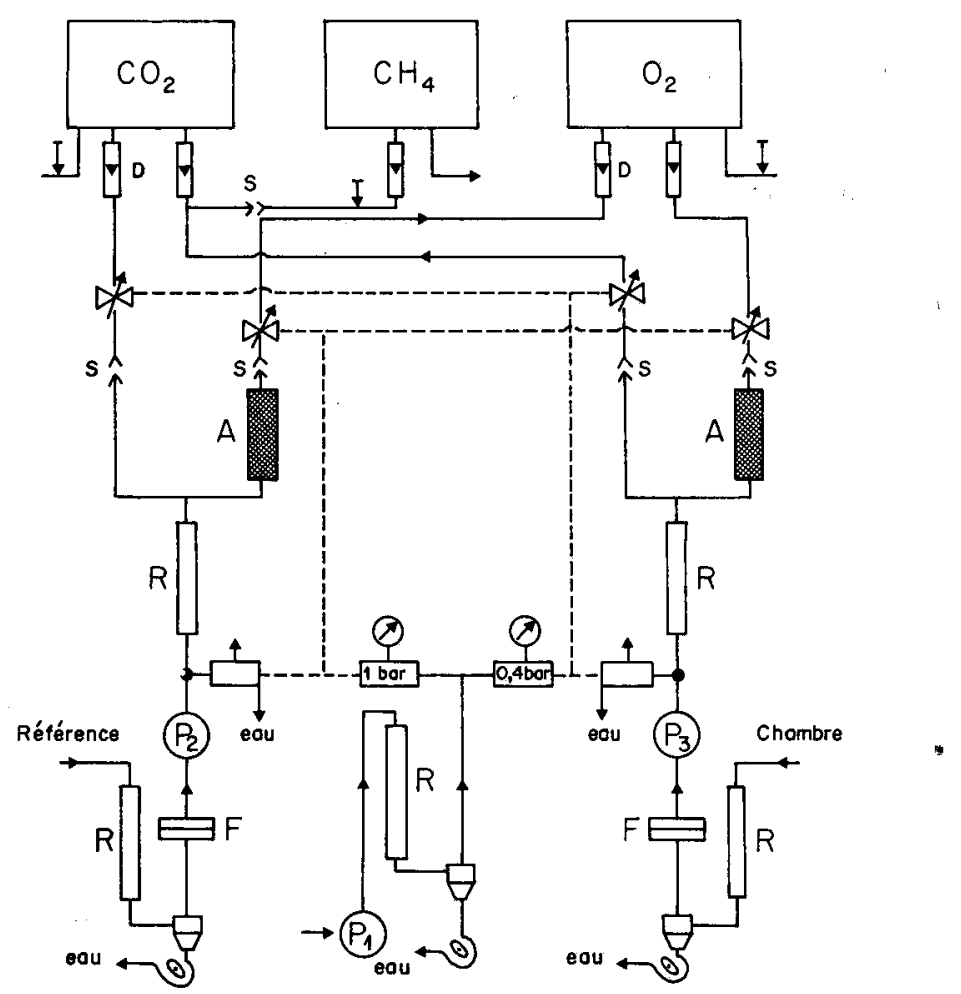

FIG. 4. - Schéma du traitement de l'air et de la régulation de pression dans la baie d'analyse

Le circuit d'une seule chambre respiratoire est représenté $\left(\mathrm{P}_{3}\right)$ $P$ : pompes ;

$\mathrm{Pl}$ : pompes assurant la régulation de pression ;

$\mathrm{R}$ : réfrigérant ;

$\mathrm{F}$ : filtre plat ;
A : actigel ;

$S$ : raccord Staubli ;

$\mathrm{D}:$ débitmètr

FIG. 4. - Airflow and pressure regulation in the gas analysis system

The circuit from one respiration chamber only is shown

$\mathrm{P}$ : pumps;

$\mathrm{P}:$ pumps régulating pressure ;

$\mathrm{R}$ : refrigerant;

A : drying agent ;

F : filter ;

$S$ : junctions:

$\mathrm{D}$ : flowmeter;

3. Les analyseurs d'oxygène, modèle Oxygor (Mahiak, Aflemagne) utilisent les propriétés paramagnétiques de l'oxygène. Contrairement aux analyseurs thermomagnétiques, le principe de ces appareils est la mesure de l'effet de pression net entre 2 gaz de susceptibilités paramagnétiques différentes (fig. 5) ce qui permet de déterminer directement la différence de teneurs en oxygène de l'air qui entre et de l'air qui sort de la chambre. L'échelle de mesure est de I p. Ioo (2I à 20 p. IOO) avec une sortie linéaire. 
L'oxygor est fortement influencé par la présence de vapeur d'eau dans l'air ; ainsi une pression partielle de $6 \mathrm{~mm}$ de vapeur d'eau environ entraîne une erreur de $7 \mathrm{p}$. Ioo. Nous avons donc dû dessécher complètement l'air, après son passage sur un réfrigérant à $3 \pm \mathrm{I}^{\circ} \mathrm{C}$, en le faisant circuler à travers du silicagel avant de pénétrer dans l'analyseur d'oxygène. Le silicagel doit être changé tous les 2 jours ; nous n'avons pas pu utiliser d'autre produit déshydratant (chlorure de calcium, sulfate de calcium, déhydrite) en raison de la formation rapide de bouchons.

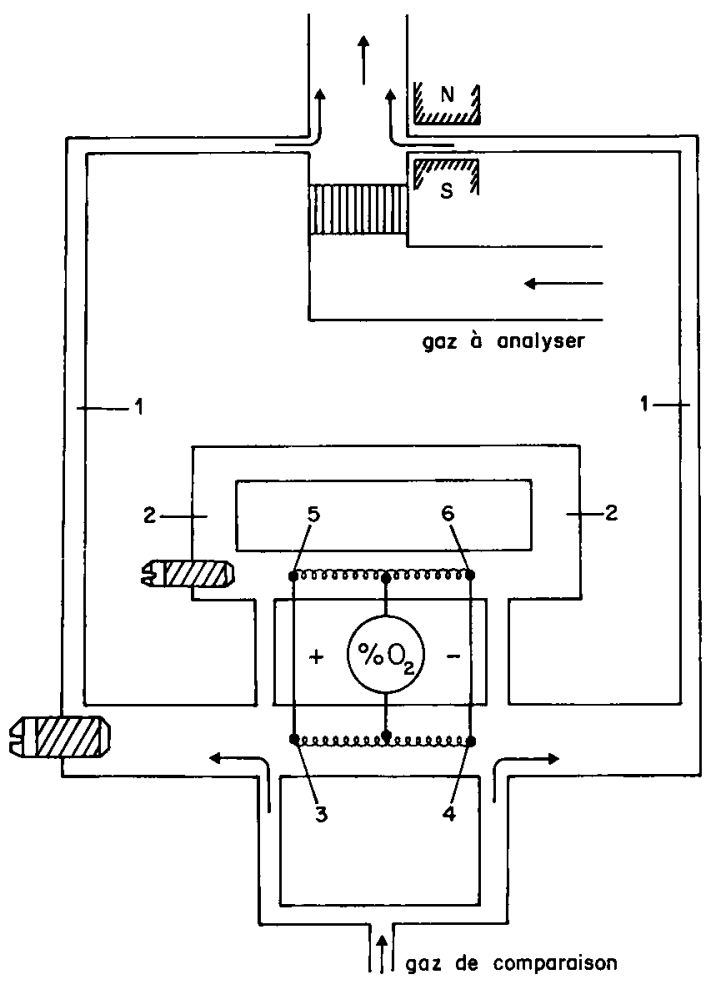

FIG. 5. - Schema de principe de l'analyse d'oxygène Oxygor

I : circuit du gaz de comparaison ;

2 : circuit secondaire du gaz de comparaison;

3-4 : détecteur thermique ;

5-6 : détecteur thermique rééquilibrant le pont de Wheatstone ;

N-S : aimant fort.

FIG. 5. - Principle of Oxygor oxygen analyzer

I : reference gas circuit ;

2 : secondary reference gas circuit ;

3-4 : " heat sensors ";

5-6: "heat sensors" balancing the Wheatstone bridge;

N-S : magnet.

Ces 3 types d'analyseurs de gaz indiquent des concentrations alors qu'ils mesurent des pressions partielles. Ils sont, de ce fait, très sensibles aux variations de pression atmosphérique. Pour remédier à cet inconvénient, nous avons équipé la baie d'analyse d'une régulation de pression (fig. 4) réglée à 400 mbars au-dessus de la pression atmosphérique du lieu (9ro \pm 25 mbars). 
4. L'analyse de l'air en continu : l'air circule en permanence à travers les analyseurs de gaz qui peuvent être scrutés avec une périodicité variable suivant le type d'expérience. Nous utilisons en routine une période de 2 minutes qui nous permet de suivre les échanges respiratoires de l'animal et d'atteindre une bonne précision. Ainsi sur une période de 24 heures on observe une différence de I p. Ioo dans la mesure des quantités d'oxygène consommé et de gaz carbonique produit pour des périodes de scrutation de 2 minutes ou de ro minutes. Cependant, au cours des premiers mois de fonctionnement, 1'installation a subi plusieurs types de pannes au niveau des analyseurs de gaz, de la centrale de mesure et de l'enregistrement des informations. Aussi avons-nous prévu un dispositif de secours qui nous permet de déterminer globalement les échanges respiratoires de l'animal au cours de la journée de mesure.

5. La constitution d'un échantillon moyen d'air : le dispositif que nous avons réalisé est comparable dans son principe à ceux utilisés et décrits par SchIEMANN (I958), VAN ES (I96I), THORBEK (I969).

Une petite fraction ( 3 à Io $\times$ IO $^{-5}$ ) de l'air qui sort de chaque chambre respiratoire est recueillie en permanence, avec un débit constant à la partie supérieure d'un cylindre en pyrex de $90 \mathrm{~mm}$ de diamètre et $\mathrm{I}, 50 \mathrm{~m}$ de hauteur (volume utile

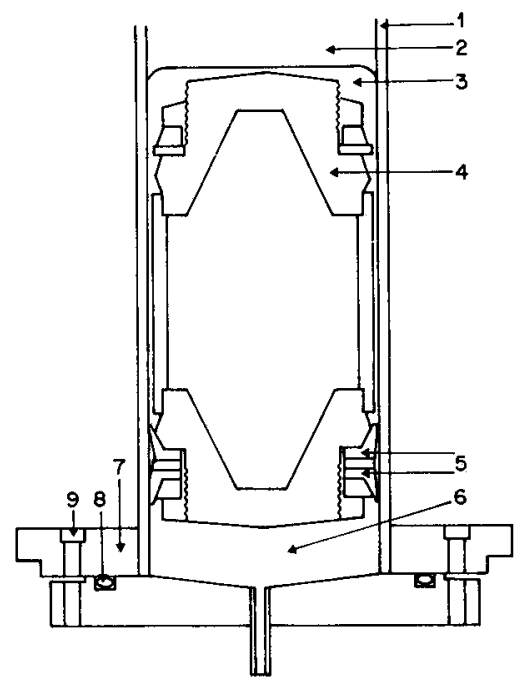

FIG. 6. - Schéma du piston et de la base du cylindre

$$
\begin{aligned}
& \text { I : tube en Pyrex; } 4 \text { : piston; } 7 \text { : base du tube } \\
& 2 \text { : air ; } 5: \text { joints; } 8: \text { joint torique ; } \\
& 3 \text { : mercure; } 6: \text { eau; } 9 \text { : vis. }
\end{aligned}
$$

FIG. 6. - Design of piston and of the base of the cylinder

$\begin{array}{lll}\text { I : Pyrex cylinder ; } & 4: \text { piston ; } & 7 \text { : base of cylinder ; } \\ 2: \text { air ; } & 5 \text { : piston rings ; } & 8 \text { : gasket; } \\ 3: \text { mercury ; } & 6 \text { : water ; } & 9 \text { : Screw. }\end{array}$

de 8 litres). Le piston en polychlorure de vinyle (fig. 6) est équipé d'un joint de plastique souple et recouvert de mercure. Son déplacement régulier est assuré par le transvasement de l'eau située à la base du cylindre. Une pompe péristaltique Sigma- 
Motor, réglée sur un débit de $0,31 /$ heure, prélève cette eau, provoque la descente du piston et le prélèvement d'air. En fin de période de mesure ( 23 à $23 \mathrm{~h} 30$ ), l'échantillon d'air est homogénéisé par convection au moyen d'une résistance électrique enroulée à la base du cylindre. Il est envoyé sous pression dans les analyseurs de gaz préalablement étalonnés, à l'aide d'une pompe Wab (Prolabo) qui injecte à la base du cylindre l'eau prélevée précédemment. Ce dispositif de secours a pu être réalisé assez rapidement au laboratoire mais demande une surveillance quotidienne.

\section{F. - Les autres capteurs physiques et les capteurs physiologiques}

\section{Les capteurs physiques.}

Les températures sont mesurées au moyen de sondes platine roo ohms à $0^{\circ} \mathrm{C}$. Pour les mesures d'humidité relative à l'intérieur de la chambre respiratoire, une petite turbine assure la circulation de l'air à une vitesse supérieure à $3 \mathrm{~m} / \mathrm{seconde}$ au niveau de la sonde sèche et de la sonde humide.

La vitesse de l'air peut être mesurée en différents points de la chambre respiratoire à l'aide d'un anémomètre Hastings dont la tête est mobile. Cet appareil utilise le principe de la thermopile et est compensé pour les variations de température ambiante. Il fournit un signal de o à 5 volts pour des vitesses variant de o à 5 mètres/ seconde. La courbe de réponse tend vers une asymptote, aussi la mesure n'est-elle précise que dans le zone de o à 2 mètres/seconde ; l'appareil peut alors servir également de débitmètre massique.

Le capteur de pression absolue (o à I ooo mba, type 437 GD, Schlumberger) est constitué d'une capsule anaéroïde dont les déformations entraînent le noyau mobile d'un transformateur différentiel. Il permet d'enregistrar les variations journalières de la pression atmosphérique.

Les capteurs physiologiques sont de deux types : des capteurs de température et des capteurs de fréquences cardiaque et respiratoire. Nous avons adapté au veau un appareil de surveillance intensive prévu pour les bébés prématurés (modèle VSM roo, Beckman). Deux électrodes biopotentielles $(\mathrm{Ag}, \mathrm{AgCl})$ de type miniature $(8 \mathrm{~mm}$ de diamètre) suffisent pour la mesure des fréquences cardiaque et respiratoire grâce à la variation d'inpédance de la cage thoracique. Ces électrodes sont insensibles aux mouvements de l'animal; elles ne sont pas affectées par l'humidité et peuvent rester en place plusieurs jours. La température rectale est mesurée à l'aide de thermistances dans la plage de 32 à $4 \mathrm{I}^{\circ} \mathrm{C}$. Des alarmes sonores et visuelles sont prévues, avec des seuils réglables, pour la période d'apnée, les fréquences cardiaque et respiratoire et les températures. Cet appareil mesure et contrôle en permanence ces différents paramètres et s'avère très utile pour les études de bioclimatologie.

\section{G. - La centralisation des mesures}

Celle-ci permet de regrouper les données des différents capteurs, d'obtenir instantanément les diverses données et de disposer de rétroactions par des consignes mises en mémoire (seuils d'alarme ou d'action) (GollLot et Treussart, I970). L'installation a été fournie par la Société Schlumberger, Diffusion internationale, Bagneux ; les différents capteurs sont scrutés à des fréquences variables et les infor- 
mations sont linéarisées puis d'une part imprimées par une machine à écrire sous forme de journal de marche et d'autre part perforées sur bande en vue de l'exploitation mécanographique (fig. 7).

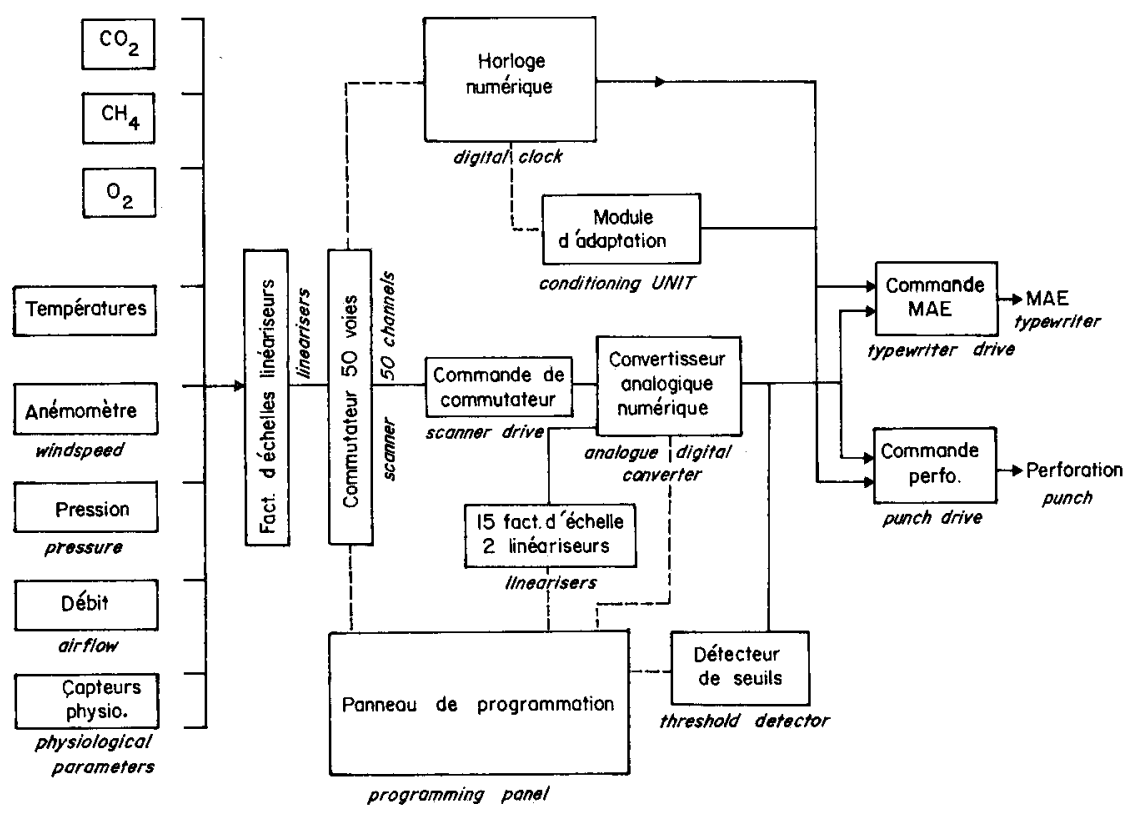

FIG. 7. - Schéma de la centrale de mesure

FIG. 7. - Scheme of central recording sysiem (see figure for translation of individual terms)

Les tensions fournies par les capteurs sont transformées en valeurs numériques par un convertisseur analogique numérique, un voltmètre intégrateur et digital Solartron ( 5 gammes de tension d'entrée, de $25 \mathrm{mV}$ à Ioo V).

I a centrale dispose d'un bloc linéariseur permettant soit d'utiliser un facteur d'échelle inférieur à $I$, dans le cas d'une sortie linéaire, pour obtenir une donnée directement utilisable, soit de linéariser une courbe par segments (exemple : analyseurs de $\mathrm{CO}_{2}$ et anémomètre Hastings). Le fournisseur a construit à notre demande un " module d'adaptation générateur d'espaces " qui permet d'enregistrer les informations des 20 premières voies à la fréquence de scrutation de l'horloge et les suivantes Io, 30 ou 60 fois moins souvent. Ce module est très utile pour limiter le nombre d'informations dans le cas où certains paramètres varient beaucoup plus lentement que d'autres.

Détecteur de seuils : la centrale de mesure comporte un détecteur qui peut recevoir ro cartes ( 3 actuellement) comportant chacune 20 seuils maxi ou mini. Ein dehors de l'intervalle compris entre ces seuils, le détecteur déclenche 2 types d'alarmes par l'intermédiaire des modules de commande correspondants : d'une part l'impression en rouge sur la machine à écrire et d'autre part la perforation de la valeur aberrante suivie d'un caractère fixe défini par la matrice à diodes de la perforatrice. Un troisième permet de déclencher une alarme sonore. 


\section{H. - L'équipement de sécurité de l'installation}

L'installation est équipée de plusieurs dispositifs de sécurité qui la protègent contre les pannes, les coupures d'alimentation électrique etc. Une alarme sonore avertit la personne logée dans le bâtiment. Les détecteurs de seuil contrôlent les températures, les débits, les concentrations de gaz carbonique et d'oxygène et les capteurs physiologiques. Par ailleurs, au niveau même des chambres respiratoires, 3 thermostats de gaine (l'un réglé à $60^{\circ} \mathrm{C}$ en aval des résistances électriques, les 2 autres dans une des gaines d'aspiration) coupant l'alimentation des résistances électriques ou la circulation d'eau froide suivant les consignes affichées et éclairent le voyant "régulation " sur le pupitre de commande.

La centrale de mesure, la baie d'analyse, les débitmètres, les capteurs physiologiques, la machine à écrire et la perforatrice de rubans sont alimentés par un chargeur convertisseur de 600 VA qui élimine les variations de tension électrique et les coupures brèves. En cas de coupure de courant secteur d'une durée supérieure à 2 minutes un groupe électrogàne alimente le chargeur-convertisseur et les turbines d'extraction.

\section{III. - CONTRÔLES DU FONCTIONNEMENT DE L'INSTALLATION}

\section{A. - La climatisation}

Pour ces contrôles nous avons simulé les productions d'eau et de chaleur d'un veau de roo à $200 \mathrm{~kg}$ à l'aide d'un humidificateur ( 250 à $500 \mathrm{~g}$ d'eau/h) et de résistances électriques ventilées dissipant de 300 à $45^{\circ}$ watts. Les performances ainsi réalisées sont représentées dans la figure 8 . Dans la plage de $\mathrm{I}_{4}$ à $28^{\circ} \mathrm{C}$ on dispose d'une grande amplitude pour l'humidité relative. Au-delà de ces températues, il faut injecter de l'eau avec la chaudière pour atteindre une hygrométrie supérieure ou égale à 90 . A ro ou $\mathrm{II}^{\circ} \mathrm{C}$, la régulation de température ne se fait que par le froid.

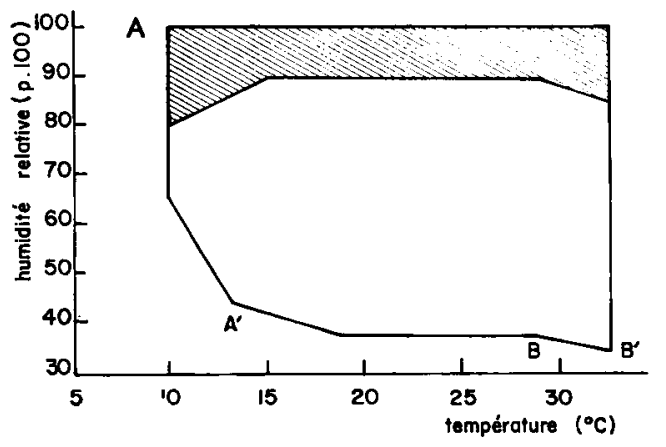

FIG. 8. - Performances du système de climatisation

Zone hachurée : avec injection de vapeur;

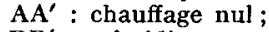

$\mathrm{BB}^{\prime}$ : refroidissement nul.

FIG. 8. - Performance of air conditioning system

Shaded a : with injection of vapour;

$\mathrm{AA}^{\prime}$ : no heating;

$\mathrm{BB}^{\prime}$ : no refrigeration. 
En effet, la température du bac froid est limitée à $2^{\circ} \mathrm{C}$ pour éviter la formation de bouchons de glace dans les réfrigérants de la baie d'analyse. De ce fait l'humidité relative minimum augmente régulièrement avec la diminution de température.

En un point donné de la chambre respiratoire, les variations de température et d'humidité relative sont inférieures ou égales respectivement à $\pm 0,2^{\circ} \mathrm{C}$ et $\pm 2 \mathrm{p}$. Ioo. La température est uniforme dans un plan horizontal et la variation est de $\pm 0,5^{\circ} \mathrm{C}$ dans le plan vertical.

La vitesse de l'air à I $\mathrm{m}$ du plancher est voisine de $0,15 \mathrm{~m} / \mathrm{s}$ dans toute la chambre sauf près de la porte d'entrée où elle atteint $0,2 \mathrm{~m} / \mathrm{s}$ à cause de la proximité du déflecteur répartissant l'air climatisé. Au milieu de la chambre, dans un plan vertical nous observons un gradient de vitesse variant de bas en haut de $0,1 \mathrm{~m} / \mathrm{s}$ à $0,2 \mathrm{~m} / \mathrm{s}$.

\section{B. - Contrôle de la production d'eau de l'animal}

Ce contrôle a été réalisé à différentes températures et une humidité relative variant de 40 à 86 p. Ioo. Des quantités connues d'eau et de chaleur sont apportées dans la chambre respiratoire à l'aide d'un humidificateur et d'une résistance électrique; on détermine d'une part la variation d'humidité relative de l'air au cours de son passage dans la chambre et d'autre part la quantité de vapeur d'eau condensée sur la batterie froide et recueillie dans le barillet. Les mesures sont faites en cours de fonctionnement sur une période de 6 à $\mathbf{~} 2 \mathrm{~h}$. L'erreur relative sur le bilan est de $2 \mathrm{p}$. roo environ pour des températures inférieures à $24^{\circ} \mathrm{C}$ mais au-delà elle est très importante en raison sans doute de la condensation d'eau dans les gaines. La précision doit être améliorée dans le cas d'une mesure sur une période de $24 \mathrm{~h}$, et, pour les températures élevées par le calorifugeage efficace des gaines.

\section{C. - Etanchéité de la chambre respiratoire}

Bien que les fuites d'air n'entraînent pas d'erreurs aussi importantes avec le " circuit ouvert " qu'avec le " circuit fermé ", surtout lorsqu'on travaille en légère dépression, nous avons voulu obtenir une bonne étanchéité des chambres respiratoires en vue des études avec des composés marqués au ${ }^{14} \mathrm{C}$. Pour ces contrôles les vannes d'entrée et de sortie d'air sont fermées et on créé une surpression ou une dépression de $20 \mathrm{~g} / \mathrm{cm}^{2}$. On laisse fonctionner le système de climatisation et au bout de 6 heures on calcule la différence de volume corrigé pour les variations de température, de pression et d'humidité relative. Les fuites ainsi déterminées sont inférieures à I 1 par heure.

\section{D. - Etalonnage des débitmètres}

Les débitmètres ont été étalonnés et sont contrôlés régulièrement par une méthode gravimétrique. Une bouteille d'air comprimé est placée sur une bascule de précision (portée maximum Ioo $\mathrm{kg} \pm 2 \mathrm{~g}$ ) et reliée au débitmètre par l'intermédiaire d'un rotamètre qui permet de régler manuellement le débit. On enregistre avec la machine à écrire les données du débitmètre toutes les 2 secondes. Lorsque le débit est stabilisé, on détermine pendant une durée de 4 à Io minutes la perte de poids de la bouteille ( $550 \mathrm{a} 700 \mathrm{~g}$ ). Le débit moyen ainsi déterminé s'exprime en litres. d'air sec, à $0^{\circ} \mathrm{C}$ et $760 \mathrm{mmHg}$, par heure. 
Après avoir vérifié la stabilité des appareils, nous les réétalonnons à la fin de chaque expérience, tous les 3 à 6 mois. Pour les contrôles de février et juillet I972, avec 18 mesures de 2,5 à $9 \mathrm{~m}^{3} /$ heure pour chaque débitmètre, les équations de régression sont les suivantes :

$$
\begin{array}{lll}
\mathrm{Y}_{1}=238,76 \mathrm{X}+\mathrm{I12} & \boldsymbol{r}=0,9997 & \sigma^{\mathbf{Y}}=40 \\
\mathrm{Y}_{2}=237,95 \mathrm{X}+33 & r=0,9996 & \sigma^{\mathbf{Y}}=45
\end{array}
$$

$\mathrm{Y}$ : débit en litres air sec, $0^{\circ} \mathrm{C}, 760 \mathrm{~mm} \mathrm{Hg} /$ heure,

$\mathrm{X}$ : tension en $\mathrm{mV}$.

L'intervalle de confiance $\left(t \cdot \sigma^{\mathrm{Y}}\right)$ est donc de $\mathrm{I}, 5 \mathrm{p}$. Ioo pour un débit de $6 \mathrm{~m}^{3} /$ heure. La dérive maximum du zéro est de 0 , Io $\mathrm{mV}$ pour $\mathrm{I} 5$ jours, ce qui correspond à une erreur maximale de 0,3 à 0,6 p. roo.

\section{E. - Contrôle des analyseurs de gaz}

Le temps de réponse des analyseurs correspondant à la déviation maximum a été déterminé en enregistrant toutes les 2 secondes les valeurs indiquées après injection d'un gaz étalon. Nous avons vérifié la linéarité du signal à l'aide d'une pompe doseuse. La stabilité des appareils a été contrôlée pendant des périodes de 24 heures à vide ou en charge avec un gaz étalon. De plus, chaque jour les analyseurs sont étalonnés, ce qui nous permet de corriger leur dérive. Nous utilisons comme référence des mélanges étalons de précision fabriqués par gravimétrie

$$
\left.\mathrm{CO}_{2}: 0,800 \pm 0,003 \text { p. } 100 \quad \text { et } \mathrm{O}_{2}: 20,20 \pm 0,005 \text { p. I00 ( }{ }^{1}\right) \text {. }
$$

Ils nous permettent de déterminer et de vérifier la composition de mélanges étalons que nous constituons au laboratoire pour les étalonnages quotidiens.

Les analyseurs de gaz carbonique sont parfaitement stables à vide et en charge ils présentent une dérive maximum de 0,5 p. Ioo par 24 heures. Leur temps de réponse est inférieur à la minute.

Avec les analyseurs d'oxygène Oxygor, la déviation totale pour une différence de concentration de 0,8 p. Ioo n'est obtenue qu'au bout de 3 à 4 minutes. Ce délai est porté à 5 ou 6 minutes par l'introduction dans le circuit d'un absorbeur d'actigel d'une capacité de $200 \mathrm{ml}$. Le remplacement de cet actigel tous les 2 jours, qui provoque une interruption du circuit d'air, nécessite une stabilisation de l'appareil pendant une quinzaine de minutes. Ce délai supplémentaire est un inconvénient pour les manipulations quotidiennes. Après plusieurs heures de fonctionnement la dérive des Oxygor à vide ou en charge est inférieure ou égale à I p. Ioo de l'échelle pendant 24 heures. Dans les conditions habituelles de travail cette dérive est en moyenne de $(r, I \pm I, 5)$ p. Ioo.

\section{F. - Temps de réponse de l'installation}

Cette caractéristique nous permet de déterminer dans quelles conditions sont enregistrées les variations des échanges respiratoires de l'animal. Pour ce contrôle nous avons établi à l'aide d'une bouteille une concentration constante en $\mathrm{CO}_{2}$ dans

(1) A notre demande, ce mélange a été fabriqué par la Société l'Air Liqnide à partir d'air deshydraté et décarbonaté et d'azote ultra pur, par pesée sous-vide d'une bouteille de $\mathbf{I}^{3} \mathrm{~m}^{3}$ à l'aide d'une balance Mettler W 8 spéciale ( $8 \mathrm{~kg}$ avec une précision de $4 \mathrm{mg}$ ). 
la chambre de l'ordre de $0,6 \mathrm{p}$. Ioo. Dans un premier essai, nous avons introduit un supplément de $20 \mathrm{~g}$ environ de $\mathrm{CO}_{2}$ en $2 \mathrm{mn} \mathrm{I} 5 \mathrm{~s}$. Nous avons enregistré toutes les 2 minutes les données de l'analyseur (fig. 9). La valeur maximum de la concentration est atteinte au bout de 2 minutes et on observe une diminution exponentielle avec une période de 30 minutes. Dans un deuxième essai, nous avons brusquement

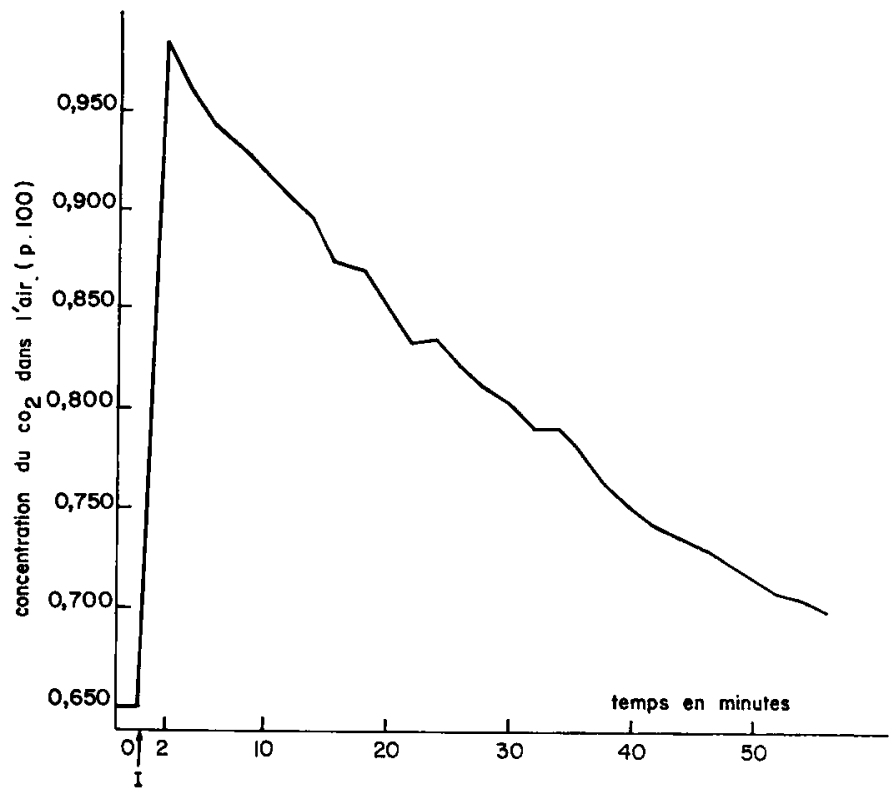

FIG. 9. - Evolution de la concentration du $\mathrm{CO}_{2}$ dans l'air de la chambre respiratoire après une injection rapide de $20 \mathrm{~g}$ de $\mathrm{CO}_{2}$

FIc. 9. - Changes in the concentration of $\mathrm{CO}_{2}$ in chamber air following the rapid injection of $20 \mathrm{~g}$ of $\mathrm{CO}_{2}$

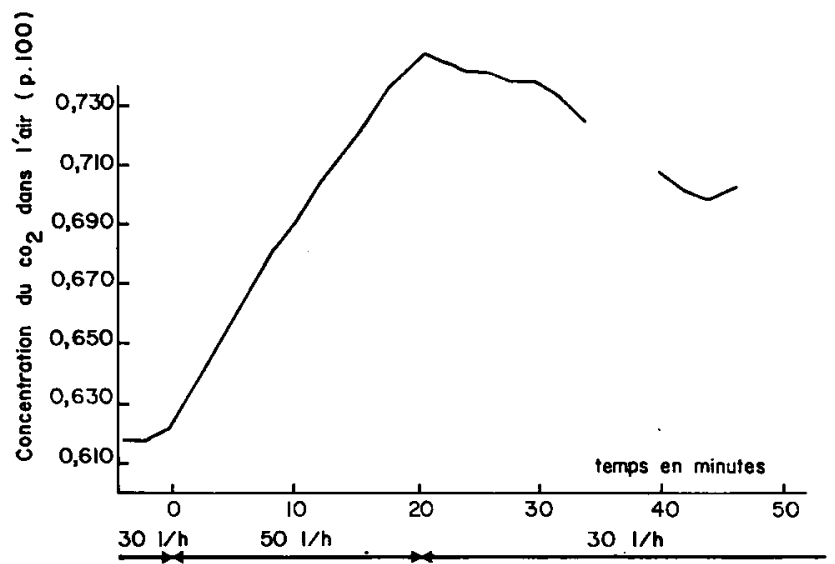

FIG. Io. - Evolution de la concentration du $\mathrm{CO}_{2}$ dans l'air en fonction de la quantitét de $\mathrm{CO}_{2}$ injectée dans la chambre respiratoire

FIG. Io. - Changes in the concentration of $\mathrm{CO}_{2}$ in air as a function of the quantity of $\mathrm{CO}_{2}$ injected into the respiration chamber 
augmenté le débit de $\mathrm{CO}_{2}$ injecté de 30 à 501 /heure pendant 20 minutes puis nous l'avons réduit à $301 /$ heure (fig. Io). Le temps de réponse est inférieur à 2 minutes et l'augmentation de la concentration est sensiblement linéaire. Ces résultats indiquent que la répartition des gaz dans la chambre respiratoire est homogène et que le temps de réponse est court pour le gaz carbonique. On peut calculer ainsi que pour une augmentation de Io $\mathrm{p}$. Ioo de la production de $\mathrm{CO}_{2}$, on observe $99 \mathrm{p}$. Ioo de la variation de la concentration en $\mathrm{CO}_{2}$ au bout de 2 minutes. De même, théoriquement, on peut mettre en évidence au bout de 2 minutes une variation de $\mathrm{I}$ p. roo de la production de $\mathrm{CO}_{2}$.

\section{G. - Contrôle de la validité de la mesure des échanges respiratoires}

Nous réalisons chaque jour un premier contrôle des quantités de $\mathrm{CO}_{2}$ produit et d'O $\mathrm{O}_{2}$ consommé en comparant les résultats donnés par 1'analyse continue de l'air d'une part et l'analyse, après étalonnage, de l'échantillon moyen d'air prélevé au cours de la journée. Les quantités obtenues avec l'échantillon moyen sont inférieures de $(\mathrm{I}, \mathrm{II} \pm 0,25)$ p. Ioo pour le $\mathrm{CO}_{2}$ et $(\mathrm{I}, 3 \pm \mathrm{I}, \mathrm{I}) \mathrm{p}$. Ioo pour l'oxygène.

Cependant la meilleure méthode de contrôle est la simulation du métabolisme de l'animal par la combustion d'un produit connu. Malheureusement la combustion d'alcool est incomplète (SchIEMANn I958, PulLar, I969) et celle du propane préconisée par Mc Graham (communication personnelle) s'est révélée irrégulière en raison probablement de la mauvaise qualité du brûleur. Aussi contrôlons-notus séparément la validité des mesures des quantités de $\mathrm{CO}_{2}$ produit et d'oxygène consommé en comparant les quantités de gaz injectées dans la chambre respiratoire vide, en fonctionnement, aux quantités calculées à partir de l'analyse de l'air et du débit.

Pour le contrôle de la mesure du gaz carbonique produit nous injectons dans la chambre du $\mathrm{CO}_{2}$ d'une bouteille de gaz comprimé à un débit déterminé pour main-

\section{TABLEAU I}

Contrôle de la validité des mesures des échanges respiratoires

Comparaison entre la quantité de gaz $\left(\mathrm{CO}_{2}, \mathrm{~N}_{2}\right)$ injectée dans la chambre respiratoire et la quantité calculée à partir des différences $(\Delta)$ de la concentrations de $\mathrm{CO}_{2}$ et $\mathrm{O}_{2}$ dans l'air et du débit de la ventilation

Test of the precision of measurement of respiratory exchange

Comparison between the quantity of $\mathrm{CO}_{2}$ and $\mathrm{N}$ injected into the respiration chamber and the amount calculated from $\mathrm{CO}_{2}$ and $\mathrm{N}$ concentration and ventilation rate

\begin{tabular}{|c|c|c|c|c|c|c|c|c|}
\hline Gaz injecté & \multicolumn{5}{|c|}{$\mathrm{CO}_{2}$} & \multicolumn{3}{|c|}{$\mathrm{N}_{3}$} \\
\hline Durée $(h) \ldots \ldots \ldots \ldots \ldots \ldots$ & 2 h 01 & $2 \mathrm{~h} 00$ & $3 \mathrm{~h} 00$ & $3 \mathrm{~h} 00$ & \& h 00 & $1 \mathrm{~h} 30$ & $2 \mathrm{~h} 20$ & $2 \mathrm{~h} 00$ \\
\hline Débit (l. air sec, $\left.0^{\circ}\right) \quad \ldots \ldots \ldots \ldots$ & 4834 & 4836 & 5129 & 5129 & 5295 & 4893 & 5060 & 10778 \\
\hline$\Delta$ moyen de concentration $(\%) \ldots$ & 0,918 & 0,902 & 0,952 & 0,943 & 0,928 & 0,935 & 0,809 & 0,933 \\
\hline Masse de gaz calculée $(\mathrm{g}) \ldots \ldots$ & 175,8 & 171,3 & 291,0 & 285,0 & 383,2 & 409,5 & 570,2 & 1200,0 \\
\hline Masse de gaz injectée $(\mathrm{g}) \ldots \ldots \ldots$ & 177 & 170 & 294 & 282 & 380 & 416 & 572 & 1186 \\
\hline Écart relatif $(\%) \ldots \ldots \ldots \ldots$ & $-0,7$ & $+0,8$ & $-1,0$ & $+1,0$ & $+0,8$ & $-1,6$ & $-0,3$ & $+1,1$ \\
\hline
\end{tabular}


tenir la concentration à 0,8 p. Ioo environ. Ensuite, nous déterminons au cours de périodes de 6 heures la perte de poids de la bouteille de $\mathrm{CO}_{2}$ et nous enregistrons en continu le débit, les températures sèche et humide de l'air et les données des analyseurs. Pour le contrôle de la mesure de l'oxygène consommé nous procédons de la même façon en utilisant une bouteille d'azote comprimé qui nous permet de diminuer la concentration d'oxygène de l'air jusqu'à 20,2 p. Ioo approximativement.

Quelques résultats de ces contrôles sont regroupés dans le tableau I. Ils montrent que dans ces conditions expérimentales, l'erreur relative est de l'ordre de I p. roo pour l'oxygène et le gaz carbonique. Compte tenu de la dérive des analyseurs sur une période de 24 heures, on peut estimer que l'erreur sur la mesure des échanges respiratoires est inférieure à $2 \mathrm{p}$. Ioo pour le gaz carbonique et de l'ordre de 2 à $3 \mathrm{p}$. roo pour l'oxygène.

\section{IV. - DÉROULEMEN'T DES EXPÉRIENCES}

\section{A. - Adaptation des animaux}

Avant leur entrée en chambres respiratoires, les veaux préruminants sont adaptés pendant 4 jours à cet environnement et au port des harnais dans des cages comparables aux cages de mesures et recouvertes d'une bâche. Ils passent ensuite 2 jours (samedi et dimanche) dans les chambres respiratoires; les principaux capteurs sont scrutés toutes les 20 minutes pour contrôler le bon fonctionnement de l'installation.

\section{B. - Mesure des échanges respiratoires}

Les mesures se poursuivent pendant 4 jours, du lundi matin au vendredi matin, la journée du vendredi étant réservée au nettoyage des cages et des chambres respiratoires et aux contrôles de routine.

Les veaux sont placés individuellement dans des cages métalliques galvanisées à roulettes. Dans une première étude relative à la valeur énergétique des fourrages, nous avons placé 2 moutons dans chaque chambre; les animaux étaient installés face à face sur un plancher recouvert d'une plaque caoutchoutée et séparés par leur mangeoire. Pour une étude sur jeunes agneaux nous avons utilisé des cages pour 7 ou 4 animaux logés individuellement. Cette solution permet d'employer les chambres respiratoires pour des études avec des animaux de petite taille (VAN Es, I97o) et de réduire la variabilité individuelle.

\section{C. - Récolte des fèces et de l'urine}

Les veaux et les moutons, toujours des mâles, sont équipés de harnais pour la collecte des fèces et de l'urine. I,es fèces sont récoltées dans un sac en polythène stocké ensuite à une température de $-\mathrm{I} 5^{\circ} \mathrm{C}$; les fèces de veau se décollent alors parfaitement. L'urine est recueillie à l'aide d'un dispositif comparable à celui décrit par WAINMAN et PATERson (I963) et aspirée en permanence dans un flacon placé à l'extérieur de la chambre, au moyen d'une pompe qui renvoie l'air dans la chambre respiratoire. Le flacon contient de l'acide sulfurique dilué pour éviter les 
pertes d'ammoniaque. Nous contrôlons une partie des pertes d'azote (MARTIN, Ig66) en dosant l'ammoniaque diluée dans l'eau condensée sur l'échangeur froid et l'azote des poils déposés sur les filtres.

\section{D. - Fonctionnement quotidien}

Le lundi matin, en cas de besoin, les chambres sont ouvertes et nettoyées vers $7 \mathrm{~h} 30$ puis refermées. Les enregistrements de mesure commencent entre $9 \mathrm{~h}$ et $9 \mathrm{~h} 30$ lorsque les concentrations de gaz ont atteint un plateau. En revanche, les autres jours les chambres ne sont pas ouvertes; pour récolter les refus, les fèces et distribuer la ration pendant la période d'arrêt des mesures, on pénètre à l'intérieur par le sas. Ce procédé évite une chute de la concentrationn en $\mathrm{CO}_{2}$ qui est longue à se rétablir à sa valeur normale. Le soir on alimente les veaux de l'extérieur à l'aide d'une pompe et les moutons en passant par le sas. Dans ce cas, le calcul montre que l'erreur est au maximum de $\mathrm{I} 1 \mathrm{de} \mathrm{CO}_{2}$ et nous n'en tenons pas compte.

Chaque matin on arrête l'enregistrement des mesures pour réétalonner les analyseurs de gaz, noter leurs dérives, et analyser les échantillons moyens d'air prélevés dans les cylindres. L'interruption dure de 30 à 45 minutes suivant le délai de stabilisation des analyseurs d'oxygène ; une personne s'occupe des analyses de gaz et de l'enregistrement des informations tandis qu'une autre donne les soins aux animaux.

$$
\text { E. - Analyses }
$$

Les échantillons de fèces et d'urine sont conservés à $-15^{\circ} \mathrm{C}$ et on constitue des échantillons moyens pour la période de collecte ( 4 jours dans le cas des veaux prérumiants). Les fèces sont lyophilisées, de même que l'urine pour les dosages d'énergie brute et de carbone. Les méthodes analytiques et la précision des résultats ont été décrites dans une publication antérieure (VERMOREL, BOUVIER, BONNET, I973).

\section{V. - EXPLOITATION DES DONNÉES}

\section{A. - Les calculs quotidiens}

Les calculs quotidiens correspondent en fait à des intégrations de données : températures, débits, échanges gazeux, paramètres physiologiques. Le nombre minimum d'informations est de 8600 par 24 heures dans le cas d'une expérience de bilan énergétique simple. Il peut être porté à 36 ooo pour une étude de bioclimatologie.

$\mathrm{L}_{\mathrm{a}}$ bande perforée est exploitée par un ordinateur PDP $8 \mathrm{E}$ équipé d'un lecteur rapide (300 caractères par seconde). On introduit manuellement les corrections de dérive des analyseurs, la durée du cycle de scrutation et le nombre de cycles à cumuler. Les différentes moyennes sont élaborées avant le calcul final. Dans les expériences de routine on imprime et perfore, pour chaque chambre et pour chaque heure de mesure, le quotient respiratoire, les quantités de $\mathrm{CO}_{2}, \mathrm{O}_{2}, \mathrm{CH}_{4}$ et le débit moyen de l'air. Un code " fin de bande " permet de calculer à la fois les valeurs correspondant à la dernière fraction d'heure, les valeurs cumulées pour la journée de mesure et le 
débit moyen en litres par minute. Cette dernière donnée est utilisée pour le calcul des échanges gazeux à partir de la composition de l'échantillon moyen d'air prélevé au cours de la journée.

Les quantités d'oxygène consommé $\left(\mathrm{O}_{2}\right)$ et de gaz carbonique produit $\left(\mathrm{CO}_{2}\right)$ se calculent par différence entre les quantités qui entrent dans les chambres respiratoires et les quantités qui en sortent, selon la relation suivante :

$$
\mathrm{O}_{2}=\left[\left(\mathrm{D}_{e} \times \mathrm{C}_{e}\right)-\left(\mathrm{D}_{s} \times \mathrm{C}_{s}\right)\right] \times t
$$

( $\mathrm{D}=$ débit, $\mathrm{C}=$ concentration).

Dans les installations du type "circuit ouvert " on ne mesure pas le débit d'entrée mais seulement le débit de sortie $\left(D_{s}\right)$ ou le volume de sortie et il y a lieu d'introduire une correction dans le calcul des échanges gazeux (VERSTEGEN et al., I97I ; McLEAN, I972). Dans notre cas nous connaissons $D_{g}$ et les différences de concentrations en $\mathrm{CO}_{2}$ et $\mathrm{O}_{2}\left(\Delta \mathrm{O}_{2}, \Delta \mathrm{CO}_{2}=\mathrm{C}_{e}-\mathrm{C}_{\varepsilon}\right)$. Soit $\mathrm{O}_{e}, \mathrm{C}_{e}$, les concentrations initiales et $\mathrm{O}_{s}, \mathrm{C}_{s}$, $\mathrm{M}$ les concentrations finales d'oxygène, de gaz carbonique et de méthane, et $\mathrm{N}_{2}$ le volume d'azote qui traverse la chambre respiratoire, on a :

$$
\begin{aligned}
\mathrm{D}_{e} & =\frac{\mathrm{N}_{2} \times 100}{100-\mathrm{C}_{e}-\mathrm{O}_{e}}=\frac{\mathrm{N}_{2} \times 100}{100-0,03-20,95}=\frac{\mathrm{N}_{2}}{79,02} \times 100 \\
\mathrm{D}_{s} & =\frac{\mathrm{N}_{2} \times 100}{100-\mathrm{C}_{s}-\mathrm{O}_{s}-\mathrm{M}} \mathrm{d}^{\prime} \text { où } \mathrm{D}_{e}=\frac{\mathrm{D}_{s}}{79,02}\left(100-\mathrm{C}_{s}-\mathrm{O}_{s}-\mathrm{M}\right)
\end{aligned}
$$

Dans ces conditions, la relation (I) devient :

- pour l'oxygène :

$$
\mathrm{O}_{2}=\left[\mathrm{D}_{\delta}\left(\frac{79,02-\mathrm{CO}_{2}-\mathrm{M}-\mathrm{O}_{2}}{79,02}\right) 20,95-\mathrm{D}_{\delta}\left(20,95-\mathrm{O}_{2}\right)\right] \times t
$$

Le calcul conduit à la formule simplifiée suivante :

$$
\mathrm{O}_{2}=\mathrm{D}_{s}\left(\mathrm{I}, 265 \mathrm{I} \Delta \mathrm{O}_{2}-0,265 \mathrm{I}\left(\Delta \mathrm{CO}_{2}+\mathrm{M}\right)\right) \times t
$$

- pour le gaz carbonique

$$
\mathrm{CO}_{2}=\mathrm{D}_{s}\left(\mathrm{I}, 0004 \Delta \mathrm{CO}_{2}-0,0004\left(\Delta \mathrm{O}_{2}+\mathrm{M}\right)\right) \times t
$$

- et pour le méthane

$$
\mathrm{CH}_{4}=\mathrm{D}_{s} \times \mathrm{M} \times t
$$

Lorsque le $Q R$ est égal à 0,7 , cette correction est de $8 \mathrm{p}$. Ioo pour l'oxygène ; pour le gaz carbonique elle est négligeable $\left(\mathrm{IO}^{-4}\right)$ et nous ne l'introduisons pas dans le programme de calcul. En revanche, nous tenons compte de la différence de composition de l'air de la chambre entre le début et la fin de la mesure; la correction est en effet, de 3,2 1 pour $0, I$ p. Ioo de différence de concentration.

Pour le calcul de la production de chaleur à partir des échanges respiratoires, nous utilisons la formule proposée par la F. E. Z. (r964)

$$
\mathrm{Q}=3,866 \mathrm{O}_{2}+\mathrm{I}, 200 \mathrm{CO}_{2}-0,5 \mathrm{I} 8 \mathrm{CH}_{4}-\mathrm{I}, 43 \mathrm{I} \mathrm{N}
$$

$\mathrm{O}_{2}, \mathrm{CO}_{2}, \mathrm{CH}_{4}$ en litres ;

$\mathrm{N}$ : azote urinaire en $\mathrm{g}$.

La production de vapeur d'eau de l'animal est la somme des quantités d'eau 
sortant de la chambre respiratoire et condensée sur la batterie froide, moins la quantité d'eau qui entre dans la chambre (VERSTEGEN, I97I). La différence de débit entre l'entrée et la sortie de la chambre respiratoire est au maximum de $0,3 \mathrm{p}$. Ioo et nous négligeons cette correction dans le calcul de la production de vapeur d'eau.

\section{B. - Le calcul des bilans en fin de période expérimentale}

Les données relatives à la durée de la période, au poids vif, aux échanges gazeux des animaux, aux quantités et aux compositions chimiques des ingesta et des excreta sont perforées sur des cartes IBM ( 2 cartes par animal). Le programme de calcul d'ordinateur IBM 7044 permet d'imprimer sous forme de tableaux facilement compréhensibles (THORBEK, I969) le poids vif et le gain de poids vif moyens de l'animal, les digestibilités moyennes de la matière sèche, de la matière organique, le bilan azoté (CUD, CUP CR), le bilan carbone, les échanges gazeux, le bilan énergétique (E. ingérée, E. digestible, EM, E fixée suivant la méthode du $Q R$ et le bilan $C-N$ ) et le pourcentage d'énergie fixée sous forme de protéines. En outre,l'ordinateur imprime et perfore sur des cartes, par $\mathrm{kg}$ de poids métabolique $\left(\mathrm{P}^{\mathbf{0}, 75}\right)$, les valeurs journalières moyennes, des quantités ingérées (matière sèche, matière organique, énergie, EM, azote) les digestibilités, la production de chaleur, les quantités d'énergie et de protéines ${ }_{s}$ ixées.

\section{C. - Exploitation statistique des résultats}

Les cartes perforées sont utilisées pour l'interprétation statistique des résultats. Cette interprétation varie avec le type d'expérience, dans le cas d'une étude de la valeur énergétique des aliments comportant plusieurs traitements (et plusieurs niveaux d'alimentation)... on fait une analyse de variance globale puis on détermine à l'aide du test de 'Tukey la variance due aux différents traitements, aux animaux, etc.

\section{D. - Contrôle de la validité des résultats, comparaison du bilan $Q R$ et du bilan $C-N$}

La comparaison des bilans obtenus par ces 2 méthodes est un contrôle de la précision des analyses et de la détermination de la consommation d'oxygène (GRAHAM et al., I958 ; HoFFMANN et SCHIEMANN, I958). En revanche, elle ne permet pas de mettre en évidence les erreurs sur les ingesta ou les excreta et sur la variation de teneur en matière sèche de ces produits.

Pour la première expérience réalisée avec des veaux de boucherie, la concordance entre les 2 bilans est très satisfaisante puisqu'en moyenne la différence est égale à $0,2 \pm \mathrm{I}, 2 \mathrm{p}$. Ioo de l'énergie ingérée.

\section{VI. - CONCLUSION}

Les 2 chambres respiratoires du type " circuit ouvert" (fig. II) qui fonctionnent depuis aôut $\mathrm{r}_{97 \mathrm{I}}$ constituent une installation complète et souple se prêtant à des études variées, nutritionnelles, métaboliques et bioclimatologiques. I,a plage de 
climatisation est assez large, la répartition de la température dans la chambre est homogène, la température et l'humidité relative sont très stables.

Ia qualité du matériel choisi a permis de réduire les inconvénients de la méthode du " circuit ouvert ": les débitmètres sont très stables et étalonnés par gra-

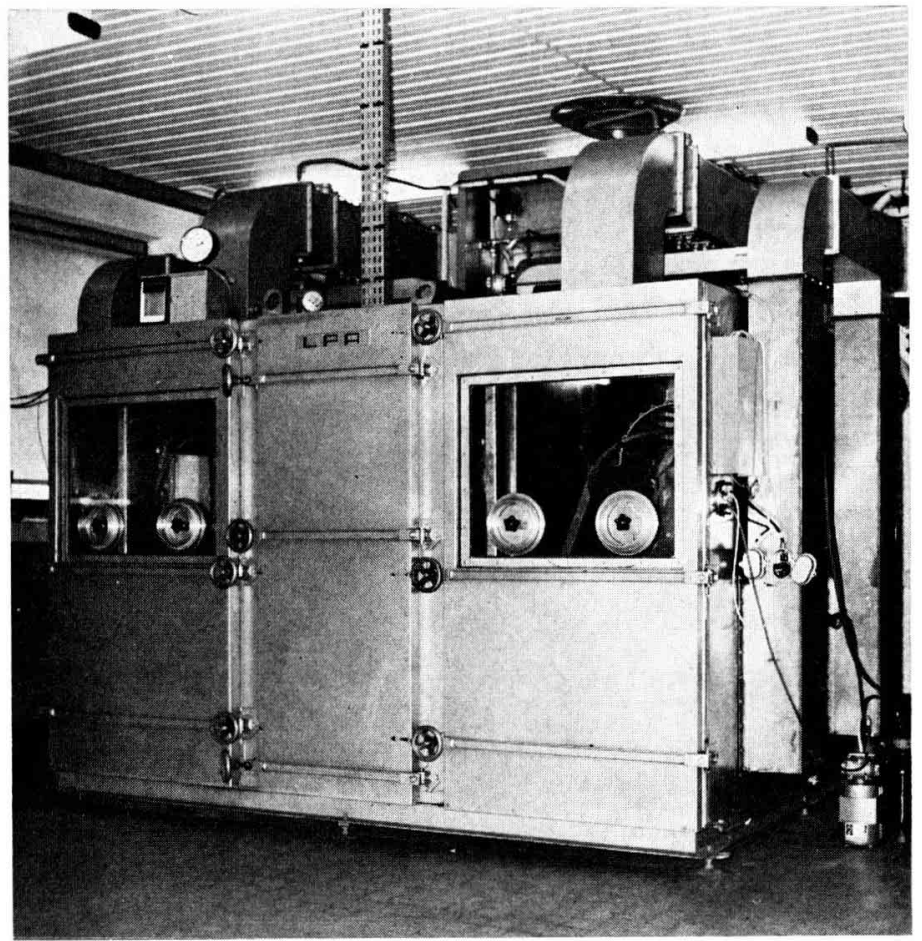

FIG. I1. - Vue d'ensemble des 2 chambres respiratoires

FIG. II. - General aspect of the two respiration chambers

vimétrie ; ils fournissent une mesure précise de la masse d'air qui a balayé les chambres respiratoires. Les analyseurs de gaz carbonique Unor (fig. I2) sont à la fois stables et précis mais dans le cas des analyseurs d'oxygène Oxygor, le délai de stabilisation, la sensibilité à la vapeur d'eau et le temps de réponse sont des sources d'erreurs à l'étalonnage. Une meilleure connaissance de ce matériel doit nous permettre d'améliorer encore la précision de nos bilans énergétiques.

L'enregistrement continu des informations avec une fréquence variable et le traitement mécanographique permettent de réaliser des études métaboliques (infusion de nutriments, influence des repas, cycles de synthèse et de mobilisation) pour lesquelles il est utile de suivre l'évolution des échanges respiratoires et du quotient respiratoire.

Ces installations se prêtent parfaitement aux études climatiques, mesure de la production d'eau de l'animal, mesures physiologiques, enregistrement fréquent des principales données, introduction possible de nouveaux capteurs. Enfin, l'adjonction de chambres respiratoires simplifiées, de petit volume, permet d'étudier l'utilisation des composés marqués au ${ }^{14} \mathrm{C}$ par le Rat ou l'Agneau. 


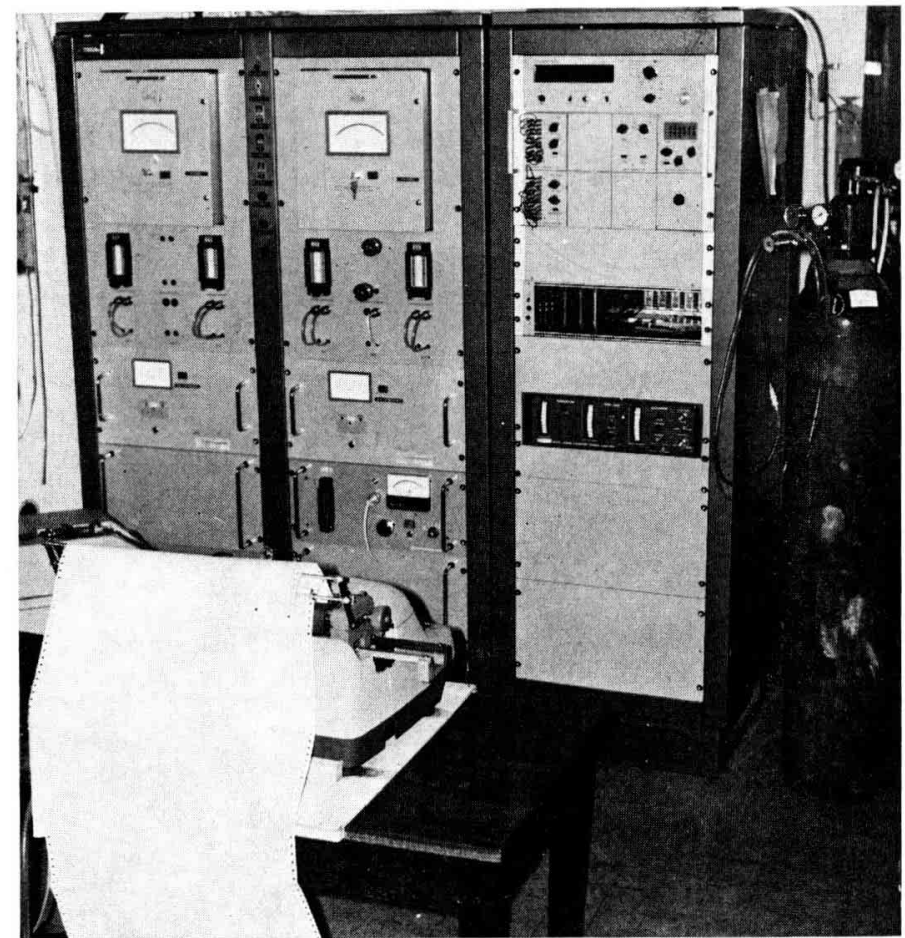

FIG. I2. - Vue d'ensemble de la baie d'analyse des gaz et de la centrale de mcsure

I: IG. 12. - General aspect of the gas analysis and central recording systems

Ces études analytiques sont pour la plupart réalisées en collaboration avec nos collègues des stations d'Élevage en complément de recherches sur la digestion ou sur l'alimentation avec des lots importants d'animaux placés dans les conditions normales d'élevage.

Reçu pour publication en mai 1973.

\section{SUMMARY}

\section{CONSTRUCTION AND OPERATION OF TWO “ OPEN CIRCUI'T " RESPIRATION CHAMBERS FOR YOUNG CATTLI}

Two respiration chambers of the "open circuit "type designed for studies in nutrition and bioclimatology have been operating since August 1971. The dimensions of the chambers (fig. I) are such that they will accommodate two yourng cattle weighing up to $350 \mathrm{~kg}$, two sheep together or groups of 7 lambs in individual digestibility cages. The two chambers are separated by an airlock and are equipped with portholes and with gloves which permit manipulation of the animals. The air conditioning system (fig. 2) can regulate temperature between ro and $32^{\circ} \mathrm{C}$ and relative humidity between 40 and $90 \mathrm{p}$. Ioo (fig. 8) with little variation $\left( \pm 0.2^{\circ} \mathrm{C}\right.$ and $\pm 2 \mathrm{p}$. IOO RH).

The flowmeters operate on the venturi principle and are equipped with microflowmeters (fig. 3) which give a linear electrical signal proportional to total flow. They are calibrated gravi- 
metrically using cylinders of compressed air. Ventilation rate can be regulated between $4^{-1} 2 \mathrm{~m}^{3} / \mathrm{h}$, with extreme stability (maximum variation $50 \mathrm{O}$ in $24 \mathrm{~h}$ ). The difference in concentration in $\mathrm{CO}_{2}$ from atmosphere is determined by two differential analyzers Unor (Mahiak) reading 0-I p. I00 ; linear and very stable. Two differential paramagnetic oxygen analyzers reading $20-2 \mathrm{I}$ p. Ioo (Oxygor) Mahiak, (fig. 5) determine the difference in concentration of oxygen between air entering and leaving the chamber; the Oxygor analyzers are very sensitive to water vapour so the air is dried over silica gel. The 4 analysers are, equipped with a pressure regulating system (fig. 4).

Air is analyzed continuously. Moreover, a support device enables the collection of a mean sample of air ( 71 ) during the $24 \mathrm{~h}$ period; a piston (fig. 6) is displaced in a vertical cylinder due to the displacement of water by a pump. The validity of the measurements of respiratory exchanges is tested by comparing quantities of gas $\left(\mathrm{CO}_{2}, \mathrm{~N}_{2}\right)$ injected into the respiratory chamber with the quantities recovered by analysis (table $\mathrm{I}$ ) ; differences are of the order of I p. Ioo. The response time of the equipment is less than $2 \mathrm{mn}$ (fig. 9 and 10 ).

The system is also equipped with physical indicators (wet and dry bulb temperatures, air movement), physiological recorders (temperatures, heat and respiration rates), threshold detectors, safety devices and alarm. These different indicators are scanned by a central recorder at a variable frequency $(\mathrm{I}, 2,10,20,30$ or $60 \mathrm{mn}$, usually $2 \mathrm{mn}$ ); the data are printed on paper (typewriter) punched onto paper tape and treated by a programme which gives mean rates per hour and totals per day. The animals spend 6 days in the respiration chamber; two days for adaptation and 4 measurement days. Energy retained and $\mathrm{C}-\mathrm{N}$ balance are calculated by computer for each period of 4 days : the difference between these two balances has been equal to $0.2 \pm \mathrm{r} .2$ p. Ioo of energy intake during the course of the first study.

This installation offers numerous possibilities for studies with animals of different sizes, in varied but well controlled climatic conditions; the continuous measurements of respiratory exchanges and physical and physiological parameters enable one to follow the progress of metabolism during the day, particularly following eating.

\section{RÉFÉRENCES BIBLIOGRAPHIQUES}

Alexander G., 196r. Energy exchanges in new-born lambs. Proc. 2th Symp. Energy Metab. Wageningen Ir-I5 september 6o-69. EAAP no ro. Laboratory of Animal Physiology. Wageningen, Netherlands.

Goillot C., Treussart H., I97o. Centralisation des mesures. Techniques d'étude des facteurs physiques de la biosphère $52 \mathrm{I}-543$. I. N. R. A. Paris.

Graham N., Blaxter K. L., Armstrong D. G., I958. Analytical and other techniques used in respiration calorimetry and their errors. Proc. $1^{\text {th }}$ Symp. Energy Metab. Copenhagen I5 I9 september 157-I63 EAAP no 8 Statens Husdyrbrugsudvalg, Copenhagen.

Graham N., I962. Influence of training and tranquillizers when measuring heat production in sheeps. Proc. Aust. Soc. Anim. Prod., 4, 138 -1 44 .

Hoffmann L., Schiemann R., I958. Der wert der Bilanzkontrolle bei Respirations versuchen. Proc. $1^{\text {th }}$ Symp. Energy Métab. Copenhagen I5-I9 sept. 234-240 EAAP n 8, Statens Husdyrbrugsudvalg, Copenhagen.

Mc Lean J. A., I972. On the calculation of heat production from open-circuit calorimetric measurements. Br.J. Nutr., 27, 597-600.

MARTIN A. K., r 966 . Some errors in the determination of nitrogen retention of sheep by nitrogen balance studies. Br.J. Nutr., 20, 325-337.

Pullar J. D., Brockway J. M., Mc Donald J. D., I969. A comparison of direct and indirect calorimetry. Proc. $4^{t h}$ Symp. Energy Metab. Warsaw sept. I967 4I5-42I EAAP ${ }^{\circ}$ I2, Oriel Press, Newcastle.

SchimmanN R., r958. Uber den Aufbau von Respirationsapparaten füı G rosstiere und Kleintiere im Oskar Kellner-Institut für Tierernährung, Rostock. Proc. $1^{t h}$ Symp. Energy Metab. Copenhagen II-I 5 sept, 29-45 EAAP n० 8, Statens Husdyrbrugsudvalg, Copenhagen.

SchneIder W., I958. Die Verwendung von automatischen Gasanalysegeräten bei Respirationsversuchen. Proc. $1^{\text {th }}$ Symp. Energy Metab. Copenhagen II-15 sept. I27-I32 EAAP n 8 , Statens Husdyrbrugsudvalg, Copenhagen.

Tноввек G., Ig69a. Studies on energy metabolism in growing pigs. I. Construction and function of a new respiration plant for pigs. Beretn fors gslab, 373, I-46.

Thorbek G., $1969 b$. Elektronische Datenverarbeitung in Stoffwechselversuchen mit Rindern, Schafen, Schweinen und Ratten. Landwirtsch. Forsch. Sonderheft, 23, 43-48.

VAN Es A. J. H., r966. Labour-saving methods for energy balance experiments with cattle : description of equipment and methods used. Neth. J. Agric. Sci., 14, 32-46. 
Van Es A. J. H., Vik-Mu L., Janssen H., Bosch A., Spreeuwenberg W., Vogt J. E., Nijkamp H. J., I97o. Balance trials with laying hens. Proc. 5th Symp. Energy Metab. Vitznan, sept. 1970, I97-201, EAAP no I 3, Juris Druck. Zurich.

Vermorel M., Bouvier J.-C., Bonnet Y., I973. Construction et fonctionnement de 4 chambres respiratoires du type "circuit fermé " pour des études de nutrition énergétique chez le Rat en croissance. Ann. Biol. anim., Bioch. Biophys., 13, 505-5I9.

Verstegen M. W. A., Van Der Hel W;, Koppe R., Van Es A. J. H., r97I. An indirect calorimeter for the measurement of the heat production of large groups of animal kept together. Meded. Labdbnvuhogeschool Wageningen, $7 \mathrm{I}, \mathrm{I} 6, \mathrm{I}-\mathrm{I} 3$.

Wainman F. W., Blaxter K. L., I958. Closed circuit respiration apparatus for the cow and steer. Developments in the design of closed circuit respiration apparatus for animals weighing up to $70 \mathrm{~kg}$. Proc. $1^{\text {th }}$ Symp. Energy Metab. Copenhagen, I I-I 5 sept, 80-95, EAAP no 8, Statens Husdyrbrugsudvalg, Copenhagen.

Wainman F. W., Paterson D., I963. A note on the collection of urine from male cattle and sheep. J. Agric. Sci., 61, 253-254. 